\title{
Relación entre contaminación atmosférica y consultas por enfermedades respiratorias en atención primaria de urgencia
}

\author{
PAULA CIFUENTES MARTÍNEZ*, ALEJANDRA RODRÍGUEZ-FERNÁNDEZ**, \\ CAROLINA LUENGO M.*** y LEONARDO TAPIA O.****
}

\section{Relationship between air pollution and respiratory disease consultations in primary emergency care}

The present investigation inquires the relation between the levels of environmental pollution by air borne particulate matter 2,5 and respiratory-related consultatons according to type of respiratory disease and age of the users who were admitted in the Primary Healthcare Emergency Services in two communes in Chile's Nuble Region, between 2016 and 2017 through an ecological design. The unit of analysis corresponded to pooled means (averages) of daily consultations for respiratory diseases and concentration of particulate matter 2.5. The statistical methods used were: Anova, statistical test Dickey-Fuller, inferential analysis based on Spearman's correlation and Cross-Correlation. A positive correlation was observed between environmental pollution and consultations related to respiratory diseases recorded the next day and the ninth day after an environmental emergency episode. Since the entry into force of the Atmospheric Prevention and Decontamination Plan, no significant differences have been observed in the concentrations of $P M_{2.5}$ between 2016 and 2017. The highest levels of environmental pollution are concentrated between the months of April to September. In conclusion, there is a relation between the levels of environmental pollution by $P M_{2.5}$, the number of respiratory diseases consultatons and the age of users. Chile.

Key words: Air pollution, Particulate matter, Respiratory Tract diseases, Primary HealthCare.

\section{Resumen}

La presente investigación indaga la relación entre los niveles de contaminación ambiental por material particulado 2,5 y consultas respiratorias según tipo de enfermedad respiratoria y edad de los usuarios que consultaron en los Servicios de Atención Primaria de Urgencia de 2 comunas de la Región de Nuble, Chile, entre los años 2016 y 2017 mediante un diseño de tipo ecológico. La unidad de análisis correspondió a medias agrupadas (promedios) de consultas diarias por enfermedades respiratorias y de concentración de material particulado 2,5. Los análisis estadísticos utilizados fueron Anova, test estadístico Dickey-Fuller, análisis inferencial basado en correlación de Spearman y Cross-Correlation. Se observó una correlación positiva entre los niveles de contaminación ambiental y consultas por enfermedades respiratorias registrados al día siguiente y al noveno día posterior a un episodio de emergencia ambiental. Desde la entrada en vigencia del Plan de Prevención y Descontaminación Atmosférica no se observan diferencias significativas en las concentraciones de $M P_{2,5}$ entre los años 2016 y 2017. Los mayores niveles de contaminación ambiental se concentran entre los meses de abril a septiembre. En conclusión, existe relación entre

* Enfermera, Magíster en Salud Pública. CESFAM Dr. Federico Puga Borne, Chillán Viejo. Chile.

** Bióloga, Magíster Estadística, Magíster Salud Pública. Profesora Asistente, Departamento de Nutrición y Salud Pública, FACSA, Universidad del Bío-Bío, Chile.

*** Enfermera, Doctora en enfermería. Profesora Asistente. Departamento de Enfermería, FACSA, Universidad del Bío-Bío, Chile.

**** Ingeniero Ambiental, Magíster en Política y Gobierno. Municipalidad de Yungay. Chile. 
los niveles de contaminación ambiental por $M P_{2,5}$ con el número de consultas por enfermedades respiratorias y la edad de los usuarios.

Palabras clave: Contaminación atmosférica; Material Particulado; Enfermedades respiratorias; Atención primaria en salud; Chile.

\section{Introducción}

La contaminación atmosférica se entiende como la presencia de sustancias contaminantes en el aire que interfieren en la salud o bienestar humano, y que producen otros efectos perjudiciales al medio ambiente. Estas sustancias pueden ser gases, material particulado o compuestos orgánicos volátiles, presentes durante lapsos prolongados y en cantidades que superen los niveles de tolerancia permitidos ${ }^{1}$. Entre los principales componentes que se depositan en las vías respiratorias y en el tejido pulmonar están partículas de metales pesados, óxidos ácidos, contaminantes orgánicos, bacterias, hongos y virus ${ }^{2}$. Las formas más dañinas de material particulado (MP) incluyen $\mathrm{MP}_{10}(\leq 10 \mu \mathrm{m}$ de diámetro aerodinámico), partículas finas: $\mathrm{MP}_{2,5}(\leq 2,5 \mu \mathrm{m})$ y partículas ultra finas $(\leq 0,1 \mu \mathrm{m})$,estas dos últimas por su tamaño $\leq 2,5 \mu \mathrm{m}$ pueden penetrar más profundamente las vías respiratorias ${ }^{3}$.

En Chile, durante los meses de invierno los índices de contaminación aumentan considerablemente debido a fuentes de emisión antropogénicas estacionarias, situación que se refleja en el área de estudio de esta investigación, cuyos niveles de contaminación atmosférica son altos en período invernal debido al intenso consumo de biomasa (leña) para uso de calefacción domiciliaria ${ }^{4}$.

Estudios internacionales previos, han informado acerca de la asociación entre contaminación del aire y salud humana, principalmente la salud respiratoria, teniendo impacto principalmente en la disminución de la función pulmonar y aumento de síntomas respiratorios, derivando a un incremento de las hospitalizaciones por patologías respiratorias ${ }^{5-7}$.

Se ha demostrado una correlación positiva entre los contaminantes del aire y la morbilidad por infecciones respiratorias virales, asma y enfermedad pulmonar obstructiva crónica ${ }^{8,9}$, debido a que el material particulado desencadena un mecanismo celular que promueve el estrés oxidativo induciendo respuesta inflamatoria de las vías aéreas ${ }^{10}$.

Según datos recopilados del Departamento de Estadística e Información en Salud del Ministerio de Salud (DEIS) en el año 2017, el 37,2\% de las consultas realizadas en los SAPU (Servicio de
Atención Primaria de Urgencia) de las comunas de Chillán y Chillán viejo correspondieron a consultas por enfermedades respiratorias (ER). Por otra parte, en el mismo año y de acuerdo a la misma fuente, en el Hospital Clínico Herminda Martin de Chillán se registraron 1.597 egresos hospitalarios por enfermedades del sistema respiratorio, representando un $8,3 \%$ del total de las 21 categorías diagnósticas monitoreadas por el DEIS $^{11}$

El decreto $\mathrm{N}^{\circ} 12$ de la legislación ambiental chilena establece que la norma primaria de calidad del aire para material particulado fino $\left(\mathrm{MP}_{2,5}\right)$ es de cincuenta microgramos por metro cúbico $\left(50 \mu \mathrm{g} / \mathrm{m}^{3}\right)$, como concentración de $24 \mathrm{~h}^{4}$. No obstante, las directrices de la OMS sobre la calidad del aire (2005) para el material particulado fino $\mathrm{MP}_{2,5}$ establecen un nivel de $25 \mu \mathrm{g} / \mathrm{m}^{3}{ }^{12}$.

Existen datos históricos en donde la norma primaria de calidad del aire para $\mathrm{MP}_{2,5}$ ha sido sobrepasada en la ciudad de Chillán, comenzando en el año 2009, en donde se registró un total de 49 días por sobre la norma con un promedio de $103 \mu \mathrm{g} / \mathrm{m}^{3}$ (concentración de $24 \mathrm{~h}$ ), el año 2010 fueron 62 días con un promedio de $118 \mu \mathrm{g} / \mathrm{m}^{3}$, el año 2011 con 23 días con un promedio de $74 \mu \mathrm{g} /$ $\mathrm{m}^{3}$, el año 2012 con 55 días promediando $123 \mu \mathrm{g} /$ $\mathrm{m}^{3}$ y por último el año 2013 con 69 días con el promedio de concentración de $24 \mathrm{~h}$ de $\mathrm{MP}_{2,5}$ más alto de $151 \mu \mathrm{g} / \mathrm{m}^{34}$.

En la Región de Ñuble a fines del año 2012, el Ministerio del Medio Ambiente declaró a las comunas de Chillán y Chillán Viejo como zonas de saturación por material particulado 10 y $2,5^{13}$, posteriormente el año 2016 fue aprobado su Plan de Prevención y Descontaminación Atmosféri$\mathrm{ca}^{5}$. Para su implementación, se consideraron las variables de impacto económico y social, con un análisis breve sobre la proyección de reducción de efectos a la salud de la contaminación atmosférica, indicando como cifra 357 casos evitados de mortalidad para el período de vigencia del plan (2016-2025) ${ }^{14}$.

En este estudio se busca evaluar la relación entre los niveles de contaminación ambiental por MP 2,5 y el número de consultas respiratorias según tipo de enfermedad respiratoria y edad de los usuarios que consultan en los Servicios de Atención Primaria de Urgencia (SAPU) en Chillán y Chillán Viejo, entre los años 2016 y 2017. 


\section{Material y Métodos}

Corresponde a un estudio ecológico, que utiliza como unidad de análisis, datos agregados acerca de las consultas por ER y de contaminación ambiental por $\mathrm{MP}_{2,5}$ en un período de 2 años. Para ello se utilizaron los registros de consultas por ER provenientes de 6 Servicios de Atención Primaria de Urgencia de las comunas de Chillán y Chillán Viejo obtenidas del Departamento de Estadística e Información en Salud (DEIS) y datos de concentración diaria de $\mathrm{MP}_{2,5}$ obtenidos del Sistema de Información Nacional de Calidad del Aire (SINCA), registrados de una estación de monitoreo ambiental del aire con representatividad poblacional (EMRP). La unidad de observación correspondió a cada día en que se registró el número de consultas por ER y concentración de material particulado.

El protocolo de estudio fue aprobado por el Comité de Bioética y Bioseguridad de la Universidad del Bío-Bío.

Los datos se analizaron mediante estadística descriptiva e inferencial considerando, prueba de Anova y correlación de Spearman. Se aplicó el test estadístico Dickey-Fuller para verificar la estacionalidad de la serie para determinar la necesidad de rezagar (o diferenciar) la serie. Se realizó un análisis inferencial basado en pruebas de Cross-Correlation (correlación cruzada) para estudiar el efecto de los días de emergencia ambiental sobre la cantidad de consultas por enfermedades respiratorias en un futuro (días posteriores) tomando las siguientes consideraciones: identificación de los días con emergencia ambiental (con mediciones $\geq 170 \mu \mathrm{g} / \mathrm{m}^{3}$ ), selección de las mediciones del día de la emergencia y el de 10 días posteriores a cada emergencia; se calcularon correlaciones cruzadas entre los niveles de contaminación ambiental (concentración) de $\mathrm{MP}_{2,5} \mathrm{y}$ el número diario de consultas por enfermedades de respiratorias. El punto de corte para decidir la existencia de correlación se basa en la fórmula ${ }^{15}$, en donde $\mathrm{n}$ es el número de observaciones $\mathrm{y}$ $\mathrm{k}$ es el retraso, en este estudio $\mathrm{n}=51 \mathrm{y} \mathrm{k}=1$, el resultado del punto de corte es 0,282.

Los análisis se llevaron a cabo utilizando el software R-Project y STATA v14.0 y se consideraron estadísticamente significativos valores $p$ inferiores a 0,05 .

\section{Resultados}

En el período de tiempo evaluado en este estudio se registraron un total de 64.063 consultas por ER, siendo los diagnósticos más frecuentes IRA (infección respiratoria aguda) alta y bronquitis aguda con 47.281 y 11.715 casos respectivamente.

En relación a los niveles más altos de contaminación ambiental, se encontraron en el período de emergencia durante los meses de mayo, junio y julio con valores promedios de $210 \pm 24,7 \mu \mathrm{g} /$ $\mathrm{m}^{3}, 223 \pm 37,9 \mu \mathrm{g} / \mathrm{m}^{3} \mathrm{y} 189 \pm 0,4 \mu \mathrm{g} / \mathrm{m}^{3}$ respectivamente. Se destaca que la concentración diaria más alta para este período fue $277 \mu \mathrm{g} / \mathrm{m}^{3}$, durante el mes de junio. El nivel de preemergencia se concentró entre los meses de abril y julio con un evento aislado en el mes de enero. La concentración de material particulado 2,5 en este período fue sobre los $120 \mu \mathrm{g} / \mathrm{m}^{3}$. Por otro lado, en el nivel de alerta (abril-septiembre) la concentración alcanzó un máximo de $109 \mu \mathrm{g} / \mathrm{m}^{3}$ (Figura 1).

El número total de consultas por enfermedades respiratorias (Figura 2, panel superior) muestra una diferencia considerable en el número de consultas a servicios de urgencia (SAPU) entre los años 2016 y 2017, en los cuales es posible apreciar ciertos comportamientos cíclicos, no tan marcados sin embargo como las diferencias en las concentraciones diarias de $\mathrm{MP}_{2,5}$ (panel inferior). Dichas concentraciones tienen un comportamiento estacional o cíclico que alcanzan los mayores niveles en los meses de invierno (Figura 2).

La correlación cruzada entre la cantidad diaria de consultas por ER y las concentraciones de $\mathrm{MP}_{2,5}$ (previas o posteriores) fue estadísticamente significativa, donde se observa que el número de consultas por ER aumentan al día siguiente (día 0 ) de un episodio de emergencia ambiental y al noveno día (día 8). El desfase de los días se debe a que la serie fue diferenciada para su análisis (Figura 3).

El total de consultas por ER fue diferente estadísticamente según nivel de contaminación $(\mathrm{p}<0,01)$. De esta forma, la mayor cantidad de consultas ocurrió en los períodos de emergencia y alerta con $149,7 \pm 119,7$ y $108,6 \pm 58,7$ casos, respectivamente. Las consultas por IRA, influenza, neumonía y bronquitis aguda, también presentaron diferencias significativas $(\mathrm{p}<$ 0,01). Para todos los casos, el mayor número de consultas por dichas enfermedades, fue en los períodos de emergencia y alerta. Sólo en período de emergencia las consultas promedio por IRA alcanzaron valores de $103,4 \pm 80,5$, bronquitis 33 $\pm 29,4$, influenza $4,1 \pm 5,3$ y neumonía $3,3 \pm 4,5$ casos respectivamente. Las consultas por crisis obstructiva bronquial, y otras causas respiratorias, no fueron diferentes en los distintos períodos de contaminación $(\mathrm{p}>0,05)$ (Tabla 1$)$. 


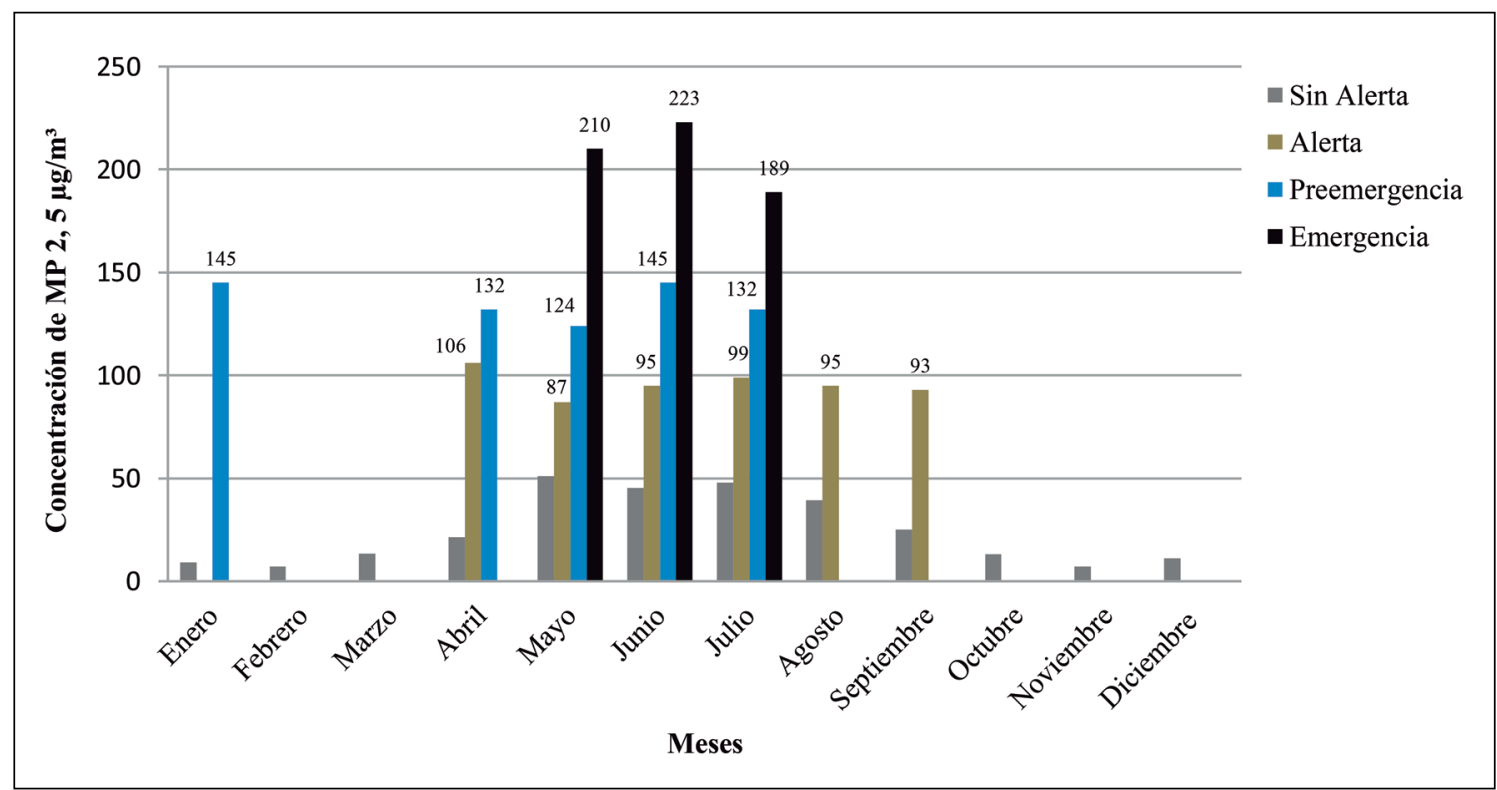

Figura 1. Gráfico de distribución de los niveles de contaminación ambiental por $\mathrm{MP}_{2,5} \mu \mathrm{g} / \mathrm{m}^{3}$, promedio mensual durante el período 2016-2017.
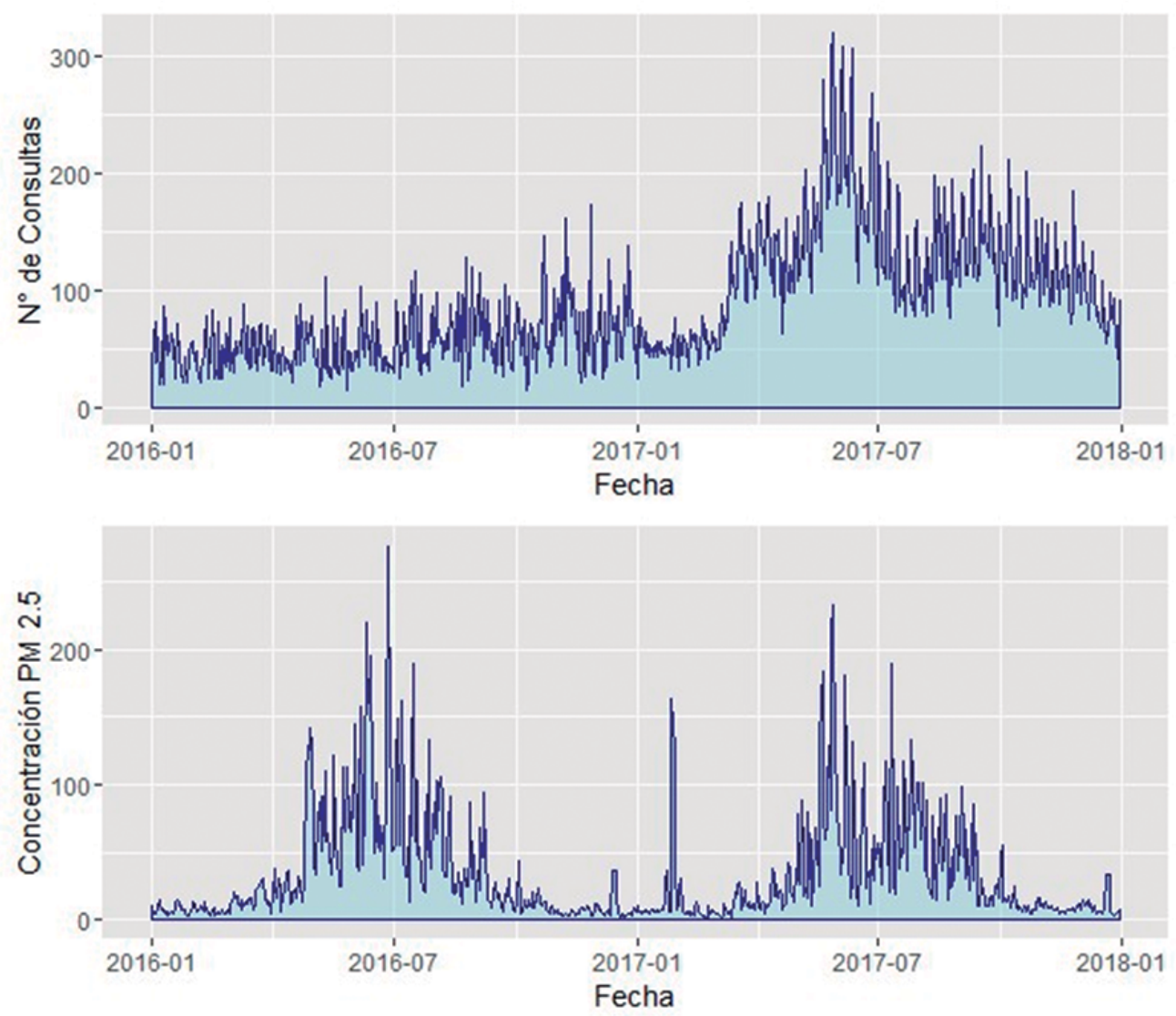

Figura 2. Panel superior: Número total de consultas por enfermedades respiratorias diarias. Panel inferior: Niveles de contaminación ambiental (concentración) diarios de $\mathrm{MP}_{2,5}$. 


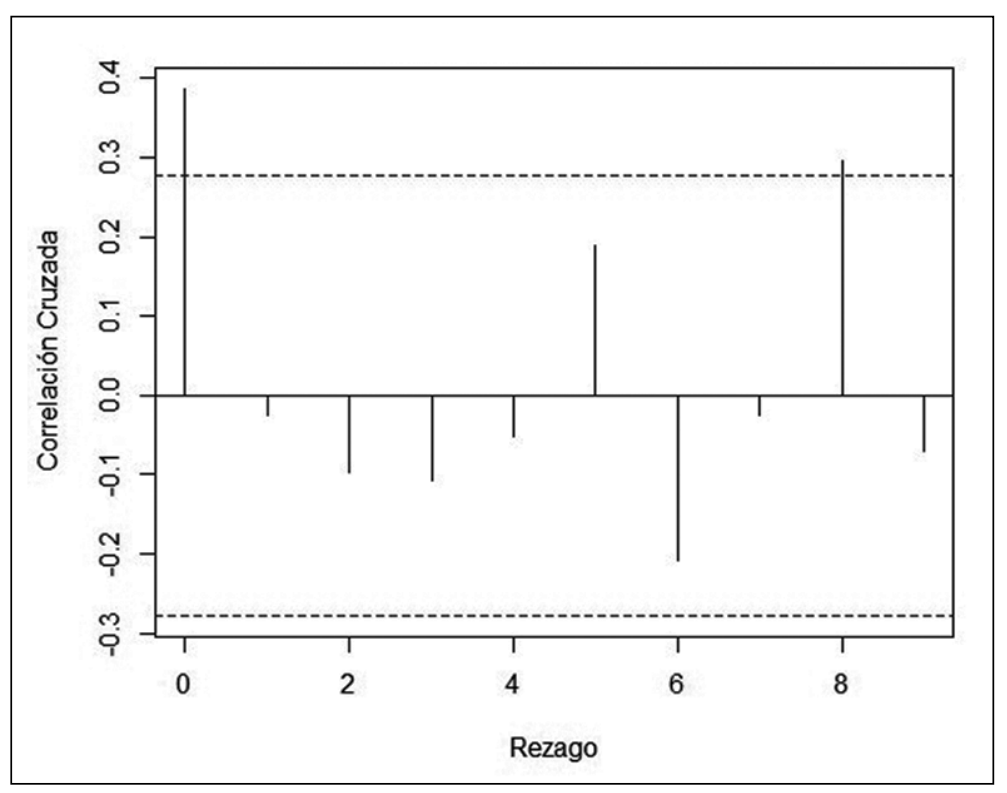

Figura 3. Función de correlación cruzada. La correlación cruzada entre la cantidad diaria de consultas por ER y las concentraciones de $\mathrm{MP}_{2,5}$ fue estadísticamente significativa.

Tabla 1. Número de consultas por enfermedades respiratorias según niveles de contaminación ambiental por $M_{2,5}$ en el período 2016-2017

\begin{tabular}{|c|c|c|c|c|c|}
\hline \multirow{2}{*}{$\begin{array}{l}\text { Consultas (n) según } \\
\text { enfermedad respiratoria }\end{array}$} & \multicolumn{4}{|c|}{ Niveles de contaminación atmosférica según $\mathbf{M P}_{2,5}$} & \multirow[b]{2}{*}{$\mathbf{p}^{*}$} \\
\hline & $\begin{array}{c}<80 \\
\text { Sin Alerta } \\
645 \text { días } \\
( \pm \text { d.e })\end{array}$ & $\begin{array}{l}\text { 80-109 } \\
\text { Alerta } \\
\text { 39 días } \\
( \pm \text { d.e })\end{array}$ & $\begin{array}{c}\text { 119-169 } \\
\text { Preemergencia } \\
35 \text { días } \\
( \pm \text { d.e })\end{array}$ & $\begin{array}{c}\geq 170\left(\mu \mathrm{g} / \mathrm{m}^{3}\right) \\
\text { Emergencia } \\
10 \text { días } \\
( \pm \text { d.e })\end{array}$ & \\
\hline Total de consultas & $85,8 \pm 49,7^{\mathrm{a}}$ & $108,6 \pm 58,7^{\mathrm{b}}$ & $85,6 \pm 51,5^{\mathrm{a}}$ & $149,7 \pm 119,7^{c}$ & $<0,01$ \\
\hline IRA Alta & $63,7 \pm 35,4^{\mathrm{a}}$ & $76,9 \pm 40,4^{b}$ & $61,8 \pm 35,8^{\mathrm{a}}$ & $103,4 \pm 80,5^{\mathrm{c}}$ & $<0,01$ \\
\hline Influenza & $1,4 \pm 2,4^{\mathrm{a}}$ & $2,1 \pm 2,6^{\mathrm{b}}$ & $2,1 \pm 3,4^{\mathrm{b}}$ & $4,1 \pm 5,3^{\mathrm{c}}$ & $<0,01$ \\
\hline Neumonía & $1,3 \pm 1,7^{\mathrm{a}}$ & $2.0 \pm 2,2^{b}$ & $1,5 \pm 1,8^{\mathrm{a}}$ & $3,3 \pm 4,5^{\mathrm{c}}$ & $<0,01$ \\
\hline Bronquitis aguda & $15,4 \pm 12,1^{\mathrm{a}}$ & $22,8 \pm 15,2^{\mathrm{b}}$ & $16,6 \pm 11,8^{\mathrm{a}}$ & $33 \pm 29,4^{c}$ & $<0,01$ \\
\hline Crisis obstructiva bronquial & $2,3 \pm 2,5$ & $3,1 \pm 2,8$ & $2,2 \pm 2,2$ & $3,6 \pm 3,6$ & 0,113 \\
\hline Otras causas respiratorias & $1,7 \pm 2,2$ & $1,6 \pm 1,5$ & $1,5 \pm 1,6$ & $2,3 \pm 2,9$ & 0,698 \\
\hline
\end{tabular}

*Valor de p según ANOVA. Con el test a posteriori de Tuckey se comparó las medias de cada fila. Las letras iguales entre dos medias indican que no hay diferencias significativas. Las letras distintas (a-b-c) entre dos medias señalan diferencias significativas entre ellas $(\mathrm{p}<0,05)$.

$\mathrm{Al}$ desagregar las consultas por ER según niveles de contaminación en diferentes rangos etarios, se observa que, para todos los rangos de edad, las consultas son mayores en el período de emergencia, las que a su vez fueron diferentes estadísticamente $(p<0,01)$. Así, las mayores frecuencias de consultas ocurrieron en los grupos etarios de 15-64 años y 5-14 años con 67,5 $\pm 54,1$ y $41,7 \pm$
38,1 casos, respectivamente. Para el grupo de 1 a 4 años el mayor número de consultas fue de 23,3 $\pm 20,3$ casos en el mismo período, al igual que en los menores de un año con 7,4 $\pm 5,8$ casos. Las consultas por enfermedades respiratorias en todos los rangos de edad fueron estadísticamente diferentes para todos los períodos de contaminación evaluados $(\mathrm{p}<0,05)$ (Tabla 2$)$. 
Tabla 2. Número de consultas por enfermedades respiratorias de grupos edad según niveles de contaminación ambiental por $\mathrm{MP}_{2,5}\left(\mu \mathrm{g} / \mathrm{m}^{3}\right)$ y períodos de emergencia ambiental en el bienio 2016-2017

\begin{tabular}{|c|c|c|c|c|c|}
\hline \multirow[t]{2}{*}{ Edad (años) } & \multicolumn{4}{|c|}{ Niveles de contaminación atmosférica según $M_{2,5}$} & \multirow[t]{2}{*}{$\mathbf{p}^{*}$} \\
\hline & $\begin{array}{c}<80 \\
\text { Sin Alerta } \\
645 \text { días } \\
( \pm \text { d.e })\end{array}$ & $\begin{array}{l}\text { 80-109 } \\
\text { Alerta } \\
39 \text { días } \\
( \pm \text { d.e })\end{array}$ & $\begin{array}{c}\text { 110-169 } \\
\text { Preemergencia } \\
35 \text { días } \\
\text { ( } \pm \text { d.e) }\end{array}$ & $\begin{array}{c}\geq 170\left(\mu \mathrm{g} / \mathrm{m}^{3}\right) \\
\text { Emergencia } \\
10 \text { días } \\
\pm \text { d.e })\end{array}$ & \\
\hline Menores de 1 & $4,4 \pm 2,9^{\mathrm{a}}$ & $5,6 \pm 3,6^{b}$ & $4,8 \pm 3,0^{\mathrm{a}}$ & $7,4 \pm 5,8^{c}$ & $<0,01$ \\
\hline 1 a 4 & $13,9 \pm 9,8^{\mathrm{a}}$ & $17,4 \pm 10,9^{b}$ & $13,4 \pm 8,3^{\mathrm{a}}$ & $23,3 \pm 20,3^{c}$ & $<0,01$ \\
\hline 5 a 14 & $17,3 \pm 12,9^{a}$ & $23,1 \pm 16,6^{b}$ & $18,5 \pm 17,9^{\mathrm{a}}$ & $41,7 \pm 38,1^{\mathrm{c}}$ & $<0,01$ \\
\hline 15 a 64 & $43,5 \pm 23,5^{a}$ & $53,9 \pm 28,9^{b}$ & $43,1 \pm 24,3^{\mathrm{a}}$ & $67,5 \pm 54,1^{\mathrm{c}}$ & $<0,01$ \\
\hline Mayores 65 & $6,7 \pm 5,4^{\mathrm{a}}$ & $8,7 \pm 6,9^{b}$ & $5,8 \pm 4,1^{\mathrm{c}}$ & $9,8 \pm 7,5^{\mathrm{d}}$ & 0,027 \\
\hline
\end{tabular}

*Valor de p según ANOVA. Con el test a posteriori de Tuckey se comparó las medias de cada fila. Las letras iguales entre dos medias indican que no hay diferencias significativas. Las letras distintas (a-b-c-d) entre dos medias señalan diferencias significativas entre ellas $(\mathrm{p}<0,05)$.

\section{Discusión}

Las concentraciones de material particulado fino $\mathrm{MP}_{2,5}$ encontrado en los años estudiados (2016 y 2017), revelan que hubo 645 días libres de niveles de alerta (inferior a $80 \mu \mathrm{g} / \mathrm{m}^{3}$ ), 39 días de alerta $\left(80-109 \mu \mathrm{g} / \mathrm{m}^{3}\right), 35$ días de preemergencia $\left(110-169 \mu \mathrm{g} / \mathrm{m}^{3}\right)$ y 10 días de emergencia (170 $\mu \mathrm{g} / \mathrm{m}^{3}$ o superior) (Tablas 1 y 2 ). Los niveles más altos de contaminación ambiental registrados (emergencia) se encuentran en los meses de mayo y junio. Los niveles de preemergencia estuvieron concentrados en los meses de abril, mayo y julio.

Por otra parte, los niveles de alerta se expresaron en un período temporal superior, presente entre los meses de abril a septiembre, correspondiente al período estacional de otoño e invierno, en los cuales la temperatura ambiental es más baja, acompañándose de aumento en el uso de calefacción intradomiciliaria en las poblaciones de ambas comunas. Preferentemente se registra un consumo intensivo de biomasa (leña) como combustible, problemática que se acrecienta al considerar las características de combustión de esta biomasa (leña húmeda) y la tecnología empleada para dicha calefacción; La sinergia de estos tres factores contribuyen a la emisión de altas tasas de material particulado fino $\mathrm{MP}_{2,5}$.

Según lo estudiado en el año 2012 por el Ministerio del Medio Ambiente, al declarar estas comunas como zonas de saturación por material particulado, se verificaron altos niveles de concentraciones de $\mathrm{MP}_{2,5}$ y $\mathrm{MP}_{10}$, observándose una marcada estacionalidad y un ciclo diario carac- terístico en donde las concentraciones promedio diarias se incrementan entre los meses de abril a septiembre ${ }^{4}$, lo cual es concordante con lo encontrado en este estudio años después (2016 y 2017).

El Plan de Prevención y Descontaminación Atmosférica de ambas comunas, que entró en vigencia el año $2016^{4}$, constituye un instrumento de gestión ambiental cuya finalidad es dar cumplimiento a la norma primaria de calidad ambiental para material particulado $\mathrm{MP}_{10}$ y $\mathrm{MP}_{2,5}$ en un plazo de 10 años. Este plan considera dos medidas estructurales: 1) el reacondicionamiento térmico de viviendas, que permitirá disminuir la demanda energética para calefacción dentro del hogar, y 2) la sustitución de los actuales sistemas de calefacción contaminantes por sistemas eficientes y con menos emisiones, que permitirá reducir las emisiones a la atmósfera y también las intradomiciliarias. Estas dos medidas se plasmaron considerando la economía local en torno a la leña, el arraigo cultural en el uso de calefacción tradicional y el problema social asociado a los costos de la calefacción.

En el Plan de Prevención y Descontaminación, también se busca resguardar la salud de la población disminuyendo el riesgo de mortalidad y morbilidad, cuyos efectos valorizados se estiman en 167 millones de dólares (USA), destacándose sus efectos en salud que dan cuenta del $90 \%$ de los beneficios del plan, principalmente por la disminución de casos de mortalidad y ahorros en gastos de atención de salud ${ }^{4}$. No obstante, dicha estimación hasta la fecha no ha sido demostrada ni documentada debido al largo plazo estable- 
cido para implementar los cambios y medidas. Sin embargo, en la Figura 2 (panel inferior), al comparar el año 2016 al 2017, es decir, a un año de la entrada en vigor del plan, no se observan diferencias significativas en las concentraciones de contaminación ambiental, por lo que la población continúa altamente expuesta al material particulado fino.

Respecto al número de consultas respiratorias luego de un día de emergencia ambiental, primeramente se logró precisar en la Figura 2 (panel superior) un aumento de las consultas por enfermedades respiratorias el año 2017 con respecto al 2016. Este hallazgo pudiera explicarse por la apertura del Servicio de Atención de Urgencia de Alta Resolución (SAR) Violeta Parra (SAPU Violeta Parra en el año 2016), dispositivo que cuenta con mayor cantidad de profesionales para resolver las consultas, con un rendimiento de atención mayor a la de un Servicio de Atención Primaria de Urgencia.

A través del método de correlación cruzada se obtuvo que posterior a un episodio de nivel de "Emergencia" $\left(\mathrm{MP}_{2,5}>170 \mu \mathrm{g} / \mathrm{m}^{3}\right)$ por concentración de material particulado fino $\mathrm{MP}_{2,5}$, se observa un aumento en la cantidad de consultas por enfermedades respiratorias, las cuales se expresan al día siguiente y al noveno día de ocurrido el episodio de emergencia. Este hallazgo se asemeja a lo encontrado el 2017 en Turquía, donde se precisó una asociación positiva entre contaminantes ambientales del aire $\left(\mathrm{MP}_{10}, \mathrm{MP}_{2,5}\right.$ y NO $\left.\mathrm{N}_{2}\right)$ $\mathrm{y}$ las admisiones hospitalarias por enfermedades respiratorias en Estambul, cuyo riesgo de padecerlas puede ocurrir hasta diez días después de la exposición a contaminación atmosférica, siendo el $\mathrm{MP}_{2,5}$ el contaminante más significativamente asociado a las admisiones por cuadros respiratorios ${ }^{16}$. Por su parte, el Plan de Prevención y Descontaminación indica a las autoridades las medidas a tomar en episodios de alerta, preemergencia y emergencia pero no menciona las directrices a ser consideradas en días posteriores: Sin embargo, los resultados sugieren la conveniencia de que los centros de atención primaria adopten medidas anticipatorias para enfrentar el siguiente día y el noveno post episodio de emergencia ambiental con un refuerzo en el personal que atiende dichos días en esas jornadas.

En resumen, se determinó la relación entre los niveles de contaminación ambiental por $\mathrm{MP}_{2,5} \mathrm{y}$ número de consultas respiratorias según tipo de enfermedad respiratoria; al respecto se encontró una correlación positiva entre niveles de contaminación ambiental con las consultas por IRA alta, influenza, neumonía y bronquitis aguda. La mayor cantidad promedio de consultas respiratorias se observó en el nivel de "emergencia". Este hallazgo se diferencia de un estudio realizado en Buenos Aires, en que si bien la mayor cantidad de consultas fue en meses de invierno, la variación en el nivel de contaminación del aire no demostró asociación significativa con las consultas del departamento de urgencias de un hospital pediátrico ${ }^{17}$.

Referente a la relación entre los niveles de contaminación ambiental por $\mathrm{MP}_{2,5} \mathrm{y}$ el número de consultas respiratorias según edad de los usuarios, se encontró una correlación positiva entre los niveles de contaminación ambiental y todos los rangos etarios estudiados, desde menores de 1 año hasta mayores de 65 años. También se aprecia que independientemente de la edad, en promedio, la mayor cantidad de consultas se registró durante episodios con niveles de emergencia.

La metodología utilizada en este estudio no permite atribuir relaciones de causalidad, sino solamente correlación entre variables, por lo tanto, se podría sostener que toda la población estaría en igualdad de condiciones de contraer una enfermedad respiratoria frente a los altos niveles contaminación ambiental. Sin embargo, existe evidencia que los niños y adultos mayores son los grupos más sensibles y vulnerables frente a la contaminación atmosférica; los niños son más susceptibles, porque necesitan inhalar el doble de aire en relación a los adultos, mientras que los ancianos se ven más afectados debido a su débil sistema inmune y a la exposición que han tenido durante su vida a contaminantes del aire ${ }^{14}$.

Se destaca la necesidad de continuar realizando este tipo de estudios en otras ciudades y comunidades de la Región de Ñuble. Una limitante importante es la no disponibilidad de estaciones de monitoreo de calidad del aire en estas ciudades.

Otra limitación de este estudio es la no disponibilidad de información del sexo de los consultantes en los Servicios de Atención Primaria de Urgencia y las restringidas categorías diagnósticas disponibles para enfermedades respiratorias. Además, al analizar rangos etarios de 15 a 64 años, no es posible precisar la diferencia entre adolescentes y adultos, limitando el análisis en poblaciones con especial vulnerabilidad, como ya se señaló.

\section{Bibliografía}

1.- WORLD HEALTH ORGANIZATION. Air pollution. Disponible en: https://www.afro.who.int/health-topics/ air-pollution. (Consultado el 10 de agosto de 2019). 
2.- GU XY, CHU X, ZENG XL, BAO HR, LIU XJ. Effects of $\mathrm{PM}_{2,5}$ exposure on the Notch signaling pathway and immune imbalance in chronic obstructive pulmonary disease. Environ Poll 2017; 226: 163-73.

3.- HYOMI K, HONGHYOK K, YOON-HYUNG P, JONG-TAE L. Assessment of temporal variation for the risk of particulate matters on asthma hospitalization. Environ Res 2017; 156: 542-50.

4.- DECRETO 48. Establece plan de prevención y descontaminación atmosférica para las comunas de Chillán y Chillán Viejo. Biblioteca del Congreso Nacional de Chile. 2015. Disponible en: https://www.bcn.cl/leychile/ navegar? $\mathrm{i}=1088773 \& \mathrm{f}=2016-03-28 \& \mathrm{p}$

5.- KIM D, CHEN Z, ZHOU LF, HUANG SX. Air pollutants and early origins of respiratory diseases. Chronic Dis Transl Med 2018; 4 :75-94.

6.- DARROW LA, KLEIN M, FLANDERS WD, MULHOLLAND JA, TOLBERT PE, STRICKLAND MJ. Air pollution and acute respiratory infections among children 0-4 years of age: an 18-year time-series study. Am J Epidemiol 2014; 180: 968-77.

7.- JACQUEMIN B, SIROUX V, SÁNCHEZ M. Ambient air pollution and adult asthma incidence in six European cohorts (ESCAPE) Environ Health Perspect 2015; 123: 613-21.

8.- DONG GH, CHEN T, LIU MM, WANG D, MA YN, REN WH, et al. Gender Differences and Effect of Air Pollution on Asthma in Children with and without Allergic Predisposition: Northeast Chinese Children Health Study. PLoS ONE 2011; 7: e22470.

9.- LIN M, STIEBD M, CHEN Y. Coarse particulate matter and hospitalization for respiratory infections in children younger than 15 years in Toronto: a case crossover analysis. Pediatrics 2005; 116: e235-e40.

10.- NENNA R, EVANGELISTI M, FRASSANITO A,
SCAGNOLARI C, PIERANGELI A, ANTONELLI G. et al. Respiratory syncytial virus bronchiolitis, weather conditions and air pollution in an Italian urban area: An observational study. Environ Res 2017; 158: 18893.

11.- MINISTERIO DE SALUD CHILE. Departamento de estadísticas e información en salud. Disponible en: Disponible en: http://www.deis.cl. (Consultado el 12 de agosto de 2019).

12.- ORGANIZACIÓN MUNDIAL DE LA SALUD. Calidad del aire y salud. 2018. Disponible en: https:// www.who.int/es/news-room/fact-sheets/detail/ambient(outdoor)-air-quality-and-health. (Consultado el 12 de agosto de 2019).

13.- DECRETO SUPREMO N³6. Declara zona saturada por material particulado respirable MP10 y por material particulado fino respirable $\mathrm{MP}_{2,5}$, ambas como concentración diaria; y declara zona latente por material particulado respirable $\mathrm{MP}_{10}$, como concentración anual, a las comunas de Chillán y Chillán Viejo. 2012.

14.- MEMORANDUM No584/2014. Análisis general del impacto económico social (AGIES) del plan de prevención y descontaminación atmosférica de Chillán y Chillán Viejo. 2014.

15.- MADHAVRAO PANDIT S, WU SM. Time Series and System Analysis with Applications. J Acoust Soc Amer 1984; 75.

16.- ÇAPRAZ Ö, DENIZ A, DOĞAN N. Effects of air pollution on respiratory hospital admissions in İstanbul, Turkey, 2013 to 2015. Chemosphere 2017; 181: 544-50.

17.- ABRUTZKY R, TORRES F, OSSORIO M, FERRERO F. Impacto de la Contaminación Atmosférica y el clima en las consultas a un departamento de emergencias pediátrico en la Ciudad de Buenos Aires. Rev Fac Cs Med. 2017; 74: 365-71.

Correspondencia a:

Paula Cifuentes Martínez

CESFAM Dr. Federico Puga Borne,

Erasmo Escala \#872, Chillán Viejo.

Región de Nuble

Email:paucifuentesm@gmail.com 